\title{
Three in one dental mirror: inovation of oral diagnostic instrument
}

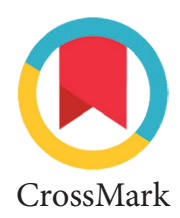

\author{
Irene E. Rieuwpassa, ${ }_{1}{ }^{*}$ Andi M. Rizal, ${ }^{1}$ Ansyari Muis, ${ }^{1}$ Harun H. Lala ${ }^{2}$
}

\section{Abstract}

Objective: This invention is to facilitate the work of the dentist in terms of examination and treatment, and increase the efficiency of time and reduce the risk of nosocomial infection.

Material and Methods: The tool that used to clean debris and water that covered the mirror surface is a dental syringe which can spray water and air. But the use of dental syringe will reduce treatment effectiveness and time efficiency, it can even increase the risk of nosocomial infection.

Results: The optimal pressure for this instrument to prevent and to clean stains on the mirror surface is between $2.5-4$ bar ( 1 bar = $0.1 \mathrm{MPa}$ ).

Conclusion: The multifunctional prototype of dental mirror which is a combination of three dental instruments in one tool has been created using the 3D printing method.
${ }^{1}$ Department of Oral Biology, Faculty of Dentistry, Hasanuddin University, Makassar, Indonesia 2Department of Mechanical Engineering, Hasanuddin University, Makassar, Indonesia

${ }^{*}$ Correspondence to: Irene $\mathrm{E}$. Rieuwpassa, Department of Oral Biology, Faculty of Dentistry, Hasanuddin University, Makassar, Indonesia drgirene@yahoo.com

Received: 19 February 2019 Revised: 25 February 2019 Accepted: 20 March 2019 Available Online 1 August 2019

Keywords: Dental mirror, Efficiency, Lamp, Syringe

Cite this Article: Rieuwpassa IE, Rizal EP, Muis A, Lala HH. 2019. Three in one dental mirror: inovation of oral diagnostic instrument. Journal of Dentomaxillofacial Science 4(2): 75-78. D01:10.15562/ jdmfs.v4i2.943

\section{Introduction}

Improvements of health degree is important in everyday life to get a healthy generation. Health problems that occur in Indonesia are increasing, especially dental and oral health. One of the dental and oral diseases that ranks highest in oral cavity is dental caries and cavities. ${ }^{1}$ Dental caries, otherwise known as tooth decay, is one of the most prevalent chronic diseases of people worldwide, it occurs in 95\% of world population. ${ }^{2,3}$ Individuals are susceptible to this disease throughout their lifetime. ${ }^{2}$ Dental caries is an infectious disease and a progressive demineralization process on the hard surface of the crown and root of the tooth that can be prevented. ${ }^{4}$ Data from Basic Health Research (RISKESDAS) in 2018 explains that the national prevalence of dental and mouth problems is $25.9 \% .^{5}$ In the treatment of dental caries and cavities, treatment must be carried out systematically and comprehensively in accordance with the principles of prevention and overall treatment. Treatment that can be done to treat caries and cavitiy is to reduce bacterial activity and prevent rapid spread by carrying out conservative treatments that are supported by the use of oral diagnostic instruments. ${ }^{6}$ Mirror is one of the oral diagnostic tools used in dentistry, especially by dentists to see the condition in the patient's oral cavity. Specific mirrors used by dentists are called dental mirrors. This tool is small, cylindrical in shape, consisting of a metal bar as a handle and a metal plate as a mirror holder.
There are 3 most important functions of the mouth mirror are retraction of the buccal and lingual soft tissues, indirect and direct visualization and illumination when used along with alight sources. ${ }^{7}$ Dental Mirror is a versatile tool in the world of dentistry, but the problem is the characteristic of conventional dental mirror that's always faced by dentists in general is mirror surface which is very vulnerable to get dirty due to the debris or water droplet. Dentists often stop treatment procedures just to clean the surface of the dental mirror so the instrument can be used again. This not only interferes with the dentist's work, but also causes the dental mirror to become unhygienic, especially if the surface is cleaned using a non-sterile cloth. At this time, a tool used for cleaning debris or water droplets in dental mirror is by using dental syringe that can spray water and air spray. However, the use of dental syringes will reduce the efficiency and working time of the dentist. This is the problem that we see as something that needs improvements. This improvement will bring benefits to dentists in terms of effectiveness and time efficiency as well as for patients in terms of reducing the risk of nosocomial infections due to unhygienic equipment. Therefore, we took the initiative to make a modification of the conventional dental mirror in the form of "Three In One Dental Mirror" which is able to prevent debris or water spray that stains the surface of the dental mirror so, that the use of the tool can be maximized without additional procedures that will take up time 
time such as cleaning the surface glass.

\section{Material and Methods}

The instrument designed in this program is a design that is made in accordance of the basic theory and specification of tool components obtained from literature studies from various sources. In fact, there are many factors that influence the percentage of success of this tool. This is why an initial design needs to be done. In the design phase of a multifunctional dental mirror, the main frame and tool components are designed. The design of the main frame and the components of the tool is carried out as a reference in the manufacture of this multifunctional dental mirror figure 1.

This three-dimensional design is needed for $3 \mathrm{D}$ printing purposes so that the tool can be printed with accuracy and precision. The operation of almost every electronic devices is concerned with electrical signals that can be either ON or OFF. ${ }^{8}$ The switch is used to turn on the lamp mounted on the appliance. Located on the outer surface of the tool handle. Lamp components is used to provide lighting when the tool is used. Located at the end of the tool leads to the mirror. This battery component is used as an energy source for LED lights. Located on the inside of the tool and wrapped by the handle of the tool figure 2 .

Components of the upper handle, where the lamp attaches to the end, a protruding switch, and the battery component inside. Components of the lower handle, a place attached to the mirror at the end, and inside there are components of the switch, battery and hose. Instrument design when all components are in place and ready to be put together. Instrument design when all components have been combined into one part figure 3 .

$3 \mathrm{D}$ printing or additive manufacturing is a process of making a three-dimensional solid object of virtually any shape from a digital model. ${ }^{9}$

\section{Results}

At the stage, manufacturing the handle component requires a high level of accuracy so that the protection of components against water and humidity can be achieved. Therefore, most of the assembly and manufacture of these tools use the help of a $3 \mathrm{D}$ printer machine. The handle of the mirror is modified so that it has space for the electrical circuit from the battery to the lamp mounted on the mirror surface. The upper surface of the syringe will be equipped with an air and water spray nozzle that is connected to the compressor using a dental hose unit. The upper surface of syringe will be equipped with an air and water spray nozzle that is connected to the compressor using a dental hose unit. The components that have been assembled and designed in such a way will then be unified to become a dental mirror unit with new modifications. Optimum pressure testing is carried out to see the water and air pressure that is suitable to use on the instrument. The lamp function test is conducted to determine whether the lamp can illuminate the oral cavity optimally.

\section{Discussion}

This instrument is made with a three-dimensional printing method (3D printer) with body material using ABS (Acrylonitrile butadiene styrene) ${ }^{10}$ and for air and water regulating valves use resin material that has a stronger strength than Polylactic Acid (PLA). ${ }^{11}$ With the concept of three-dimensional printing (3D printer) this tool has the advantage of being easy to develop using aluminum material with sand molding techniques. The specifications of this tool that is using a current of $1 \mathrm{~A}$ (amperes) with a voltage of $3 \mathrm{~V}$ (volts) so it is safe to operate in the oral cavity and the material used is made of PLA and resin that does not conduct electric current. A current of $1 \mathrm{~A}$ (amperes) and a voltage of $3 \mathrm{~V}$ (volts) allows the tool to be used for three months. In addition, this tool is also non-permanent so easy to do repairs when one component of the tool is damaged.
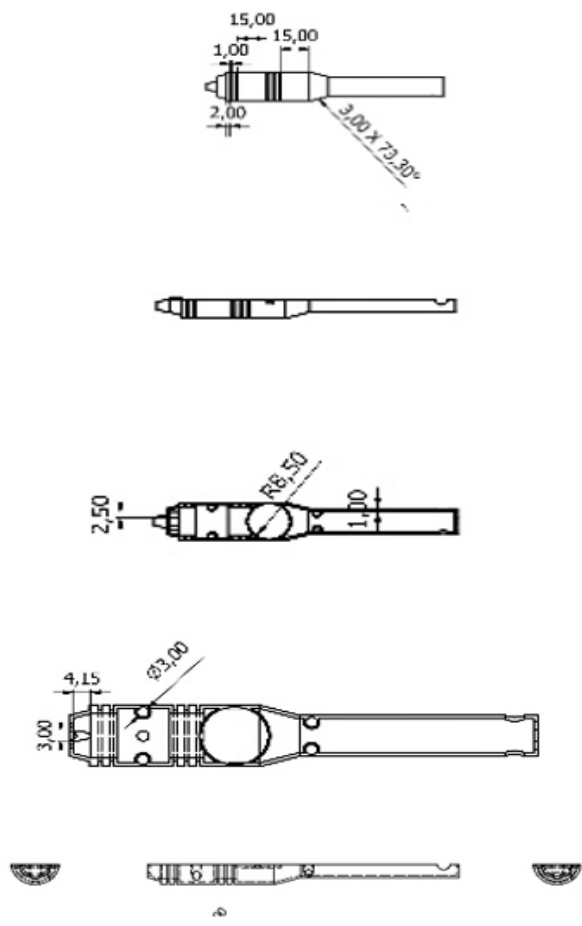

Figure 1 Lower handle design, upper handle design, unified handle design 

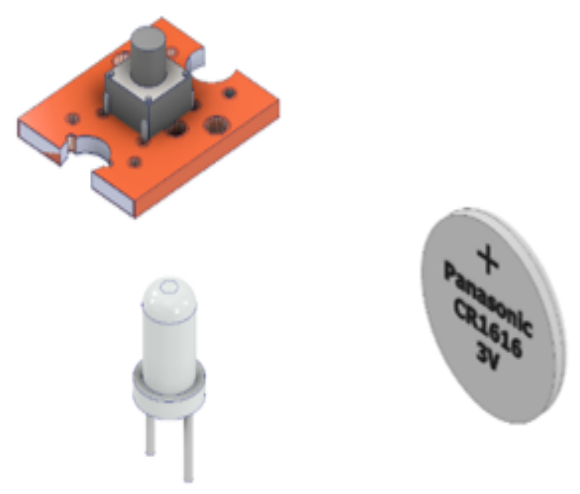

Figure 2 Switch, LED Lamp, Battery
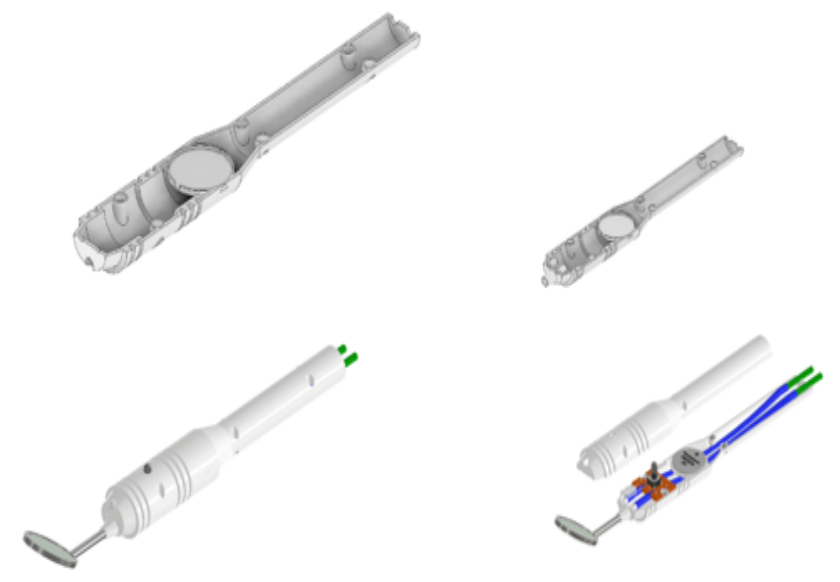

Figure 3 Upper and lower handle design and design of instruments

This instrument is a combination of three dental instruments which are put together as one instrument so that it has three functions. The function of this tool specifically is to see the condition of the oral cavity which is difficult to see directly which is equipped with an operator lamp component to provide illumination in the oral cavity as well as a syringe component to clean and rinse mirror surfaces that are affected by debris (treatment residual) or splashes of water droplets. ${ }^{12}$

Improving the effectiveness and efficiency of the work of the dentist because the syringe and operator lamp components used today are still separated from the mirror component so that in their use the dentist often stops treatment. Therefore, with this innovation, dentists can regulate lighting and manage rinsing and cleaning glass without having to stop treatment procedures.

This innovation greatly facilitates rinsing and cleaning the surface of the mouth glass because air and water valve components are placed on the foot pedal. This will greatly facilitate the dentist in cleaning and rinsing the surface of the glass so that it can reduce the possibility of cross-infection ${ }^{13}$ due to cleaning and rinsing the surface of the dental mirror using your hands/cloth.

\section{Conclusion}

The multifunctional prototype of dental mirror which is a combination of three dental instruments in one tool has been created using the 3D printing method. From the test results of the function of each component, this tool has been able to overcome conventional dental mirror problems including tools that have been able to provide lighting in the oral cavity and the tool has been able to clean and rinse a dirty mirror due to debris (treatment residual stain) or water droplet.

\section{Acknowlegment}

Thank you Faculty of Dentistry Hasanuddin University for the provided support and all involved in this research.

\section{Conflict of Interest}

The authors report no conflict of interest

\section{References}

1. Anwar AI. Correlation between mental health and caries status in primary school students. J Dentomaxillofac Sci 2018;3: 108.

2. Selwitz RH, Ismail AI, Pitts N. Dental Caries. J Lancet 2007;369: 51.

3. Mital P, Mahta N, Saini A, et al. Recent advances in detection and diagnosis of dental caries. J Evolution Med Dent Sci 2016;3: 177.

4. Surathu N, Nasim I. Assesment of the use of a dental mirror. JDMS 2015;14: 115.

5. Obot EP, Asuquo UE, Oku DE. Construction and operation of a clap light switch. IJSER 2015;6: 1983.

6. Mpofu TP, Mawere C, Mukosera M. The impact and application of 3D printing technology. ISJR 2014;3: 2148.

7. Tasdemir M. Properties of acrylonitrile butadiene styrene. J App Polymer Sci 2004;93: 2521.

8. Aguirre EC, Franco FI, Samsudin H, et al. Polylactic acid mass production, processing, industrial application, and end of life. J Adv Drug Delivery Review 2016;107: 1.

9. Shah Ah, Wyne AH. Cross infection control in dentistry: a review. Pakistan Oral Dent J 2010;30: 168.

10. Ozdemir D. Dental caries and preventive strategies. J Educ Instructional Stud 2014;4: 20.

11. Relvas M, Diz P, Seoane J, et al. Oral health scales: design of an oral helath scale of infectious potential. Med Oral Patol Oral Cir Bucal 2013;18: e664-e670.

12. Dimashkieh MR. A reverse-angle dental miror. J Prosthet Dent 2002;87: 345-346.

13. Albandar JM. Epidemiology and risk factors of periodontal diseases. Dent Clin North Am 2005;49: 517-532.

14. Chalmers JM, King PL, Spencer AJ, et al. The oral health assessment tool â validity and reliability. Aust Dent J 2005;50:191-199. 
15. Greene JC, Vermillion JR. The simplified oral hygiene index. J Am Dent Assoc 1964;68: 7.

16. Kay E, Locker D. A systematic review of the effectiveness of health promotion aimed at improving oral health. Community Dent Health. 1998;15:132-144.

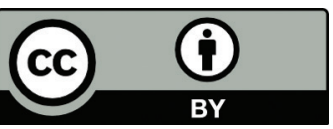

This work is licensed under a Creative Commons Attribution 